\title{
Prevalência e fatores associados ao consumo de queijo não pasteurizado entre pacientes com tuberculose de uma área urbana do Brasil*
}

\section{Prevalence and associated factors to unpasteurized cheese consumption in patients with tuberculosis in an urban area of Brazil}

\author{
Bernardo Barbosa Rocha, ${ }^{* *}$ Marcio Roberto Silva, ${ }^{* *}$ Guilherme Nunes de Souza, ${ }^{* *}$ \\ Maria Aparecida Scatamburlo Moreira, ${ }^{* * *}$ Letícia Scafutto de Faria ${ }^{\star * * * *}$
}

\section{Resumo}

No Brasil o consumo de leite cru e derivados lácteos elaborados a partir de leite cru é responsável por $30 \%$ da produção total. Um estudo de corte transversal foi realizado para estimar a prevalência e, adicionalmente, avaliar fatores associados ao consumo de queijo feito com leite cru em pacientes com tuberculose na região de Juiz de Fora, Minas Gerais. Foram construídos modelos explicativos de regressão linear univariado e multivariado. A taxa de consumo atual de queijo feito com leite cru encontrada foi de $30,7 \%$. A mediana de consumo foi de 279 unidades de queijo feito com leite cru por indivíduo ao longo da vida. As variáveis sexo, idade e ocupação relacionada com a pecuária ou agroindústria de alimentos de origem animal apresentaram-se associadas $(p \leq 0,05)$ ao consumo de queijo feito com leite cru. Os resultados reforçam a necessidade de medidas efetivas de controle do comércio de queijo feito com leite cru e de programas de conscientização dos consumidores sobre os riscos de consumo de lácteos elaborados a partir de leite cru, assegurados pelas autoridades de saúde e agricultura. Esse estudo objetivou estimar a prevalência atual ou passada de consumo, bem como as quantidades de ingestão ao longo da vida, de queijo feito com leite cru e os fatores de risco associados a esse consumo.

Palavras-chave: inspeção de alimentos, lácteos, não pasteurização, tuberculose, zoonoses.

\begin{abstract}
In Brazil the consumption of unpasteurized milk and dairy products accounted for $30 \%$ of total production. A cross-sectional study was conducted to estimate the prevalence and additionally assess factors associated to unpasteurized cheese consumption in patients with tuberculosis in the region of Juiz de Fora, Minas Gerais. Explanatory univariate and multivariate linear regression models were constructed. The current consumption rate of unpasteurized cheese was $43.9 \%$. The median of consumption was 279 units of unpasteurized cheese per individual over the lifetime. Sex, age and occupation related to farming or agribusiness of foodstuffs of animal origin, were associated $(p \leq 0.05)$ to unpasteurized milk consumption. The results reinforce the need for effective measures to control informal cheese trade, highlighting the need of health and agriculture authorities assure educational programs focusing health hazards of drinking raw milk. This study aimed to estimate the prevalence of current or past consumption, as well as the amounts of intake throughout life, cheese made with raw milk and the risk factors associated with consumption.
\end{abstract}

Keywords: dairy, food inspection, non-pasteurization, tuberculosis, zoonoses.

\section{Introdução}

Da produção total, somente 20.975 .501 dos 30.715 .460 litros de leite produzidos no Brasil passaram por algum órgão de inspeção, o que sugere que $33 \%$ do leite produzido no Brasil são consumidos pelo mercado informal, sem qualquer fiscalização higiênico-sanitária (Zoccal, 2012). Grande parte deste leite informal é destinada à fabricação de queijos artesanais elaborados a partir de leite recém-ordenhado, não pasteurizado e não fervido, ou seja, leite cru.
Essa porcentagem de leite e derivados informais torna-se maior no meio rural, em cidades de pequeno porte e periferias de médias e grandes cidades. Um estudo realizado em áreas periurbanas de Juiz de Fora, Minas Gerais, revelou taxas de consumo de leite e queijo não inspecionados de $42,0 \%$ e $66,5 \%$ das residências, respectivamente (Silva et al., 2005).

Agentes zoonóticos como Mycobaterium bovis, Brucella abortus, Campylobacter jejuni, Listeria monocytogenes, Clostridium perfringens, entre outros são transmitidos a humanos por meio da ingestão do leite e derivados crus.

\footnotetext{
*Recebido em 9 de outubro de 2013 e aceito em 29 de junho de 2014.

**Programa de Pós-Graduação em Medicina Veterinária (Mestrado) - Medicina Veterinária Preventiva e Saúde Pública - Faculdade de Veterinária Universidade Federal de Viçosa.

${ }^{* * *}$ Centro Nacional de Pesquisa em Gado de Leite - Embrapa Gado de Leite - Pesquisador A. Rua Eugênio do Nascimento, 610 - Dom Bosco, CEP. 36038-330 - Juiz de Fora - MG Tel.: (32) 33117400

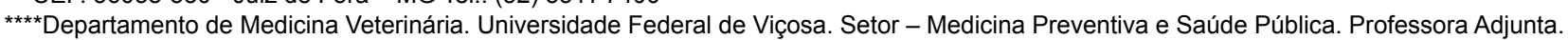

*****Programa de Pós-Graduação em Ciência e Tecnologia de Leite e Derivados (Mestrado) - Faculdade de Farmácia - Universidade Federal de Juiz de Fora. Autores para correspondência. E-mail: bernardomedvet@hotmail.com e marcio-roberto.silva@embrapa.br
} 
Em Pernambuco foi realizado um estudo para diagnóstico da brucelose em humanos. Foi encontrada uma frequência de 12 $(21,4 \%)$ pessoas sororreagentes para pesquisa de $B$. abortus (soro aglutinação lenta) em 56 pessoas analisadas; 44 (78,6\%) e $50(89,3 \%)$ delas consumiam leite cru e derivados de leite cru, respectivamente (Tenório et al., 2008).

Um estudo realizado também em Juiz de Fora, Minas Gerais, com a mesma população do presente estudo, encontrou que em 189 pacientes humanos diagnosticados com tuberculose em dois centros de referência, três $(1,6 \%)$ apresentaram evidências de infecções por $M$. bovis. Os casos de tuberculose zoonótica apresentaram associação significativa com consumo de queijo feito de leite cru (Silva et al., 2013). Os resultados encontrados alertam para os potenciais riscos à saúde da veiculação de micro-organismos zoonóticos pelo leite e derivados não pasteurizados.

Entretanto, no Brasil, há poucos estudos que visam estimar o consumo de lácteos não pasteurizados e os fatores a eles associados. Conhecer os fatores associados ao consumo de lácteos informais pode orientar a implementação de políticas públicas que visem à inibição da comercialização deste tipo de alimento.

Dessa forma, o presente estudo teve o objetivo de estimar a prevalência atual ou passada de consumo, bem como as quantidades de ingestão ao longo da vida, de queijo feito com leite cru e os fatores de risco associados ao consumo desse alimento, em populações de alta vulnerabilidade social (pacientes com tuberculose) de Juiz de Fora, Minas Gerais.

\section{Material e método}

\section{População e período do estudo}

A população do estudo foi recrutada a partir de dois centros de referência em tratamento da tuberculose de Juiz de Fora, Minas Gerais, cidade de aproximadamente 500 mil habitantes e a maior parte da população $(98 \%)$ residindo no meio urbano na atualidade. Os centros de referência incluídos foram: Hospital Reginal João Penido/Fundação Hospital do Estado de Minas Gerais e Unidade das Clínicas Especializadas do Sistema Único de Sáude. O período do estudo foi de março de 2008 a fevereiro de 2010.

Uma amostra randomizada de 189 indivíduos diagnosticados com tuberculose foi incluída no presente estudo, considerando-se uma população finita de 540 pacientes com tuberculose, uma proporção atual de consumo de queijo feito com leite cru de 30,7\%, um erro relativo de $30,7 \%$ e um nível de significância de 0,05 .

\section{Dados éticos}

Os participantes foram esclarecidos sobre os objetivos do estudo e um Termo de Consentimento Livre e Esclarecido foi obtido de todos eles, sendo o estudo aprovado pelo Conselho de Ética em Pesquisa da Universidade Federal de Juiz de Fora (protocolo 819.125.2006) e Hospital Regional João Penido (protocolo 52/08).

\section{Coleta de dados}

Após a exposição dos objetivos do estudo, foi realizada uma entrevista, utilizando-se um questionário estruturado, para coletar informações sobre as variáveis comportamentais, padrões de consumo de produtos lácteos e tipos de ocupação. A forma de consumo do queijo (feito com leite fervido, pasteurizado ou cru) foi também determinada.

\section{Delineamento do estudo}

Foi realizado um estudo de corte transversal para estimar a prevalência de consumo de queijo feito com leite cru e as quantidades de consumo deste tipo de alimento ao longo da vida.

\section{Variáveis do estudo}

O estudo teve como variável resposta (evento) o número de dias de consumo de queijo feito de leite cru ao longo da vida. A definição de queijo feito com leite cru utilizada no presente estudo refere-se ao queijo consumido elaborado a partir de leite não pasteurizado ou não fervido. O número de dias de consumo de queijo feito com leite cru ao longo da vida foi estimado a partir de perguntas abrangendo a freqüência e o período de consumo deste tipo de alimento. O número de dias de consumo de queijo elaborado a partir de leite cru ao longo da vida foi estimado a partir da frequência e o período de consumo deste tipo de alimento, construindo-se uma estimativa numérica (dias de consumo), seguindo a fórmula:

$D C=F \times P$

Onde:

$\mathrm{DC}=$ Dias de consumo;

$\mathrm{F}=$ Frequência semanal;

$\mathrm{P}=$ Período de consumo (semanas).

As possíveis variáveis explicativas do estudo foram sexo, idade, nível de escolaridade, renda familiar, local de residência, histórico de residência no meio rural, tipo de moradia, ocupação e histórico do consumo de álcool no último ano. As explicativas foram classificadas como categóricas e/ou numéricas de acordo com as análises realizadas.

\section{Análises estatísticas}

A partir das informações coletadas foi elaborado um banco de dados contendo todas as variáveis do estudo. O software Epi Info versão 3.5 .3 foi usado para gerenciamento e análise dos dados (Dean, et al., 2011 a).

Foram realizadas análises descritivas das variáveis qualitativas e quantitativas. Adicionalmente, utilizou-se modelos de regressão linear univariados e multivariados para avaliar a relação entre variáveis preditivas e o número de dias de consumo de queijo feito com leite cru. O nível de significância considerado foi de 0,05 . A variável resposta (número de dias de consumo de queijo feito com leite cru) necessitou de uma transformação logarítmica para que esta atendesse aos princípios da normalidade e homocedasticidade.

A análise de regressão linear multivariada buscou a associação independente entre variáveis explicativas e o número de dias de consumo de queijo feito de leite cru ao longo da vida. As variáveis que foram associadas ao número de dias de consumo de leite cru e queijo feito de leite cru na análise de regressão linear univariada com nível de significância $p<0,20$ e todas aquelas com relevância biológica ou epidemiológica foram consideradas para as análises de regressão linear multivariada usando o método "backward". 
Análises gráficas dos resíduos bem como alguns testes complementares foram realizadas para avaliar o nível de ajuste dos modelos. Utilizou-se o pacote Global Validation of Linear Models Assumption (GVLMA), do software R (Development Core Team, 2005), que avalia os seguintes pressupostos dos modelos: independência, linearidade, curtose, skewness e homocedasticidade. Testes complementares como ShapiroWilk e Kolmogorov-Smirnov foram realizados para avaliar a normalidade do modelo de regressão linear multivariado.

Como foi realizada transformação logarítmica da resposta $(y)$, para interpretação dos coeficientes gerados nas análises de regressão univariada a multivariada em termos originais foi feita a exponencial de $y$.

A interpretação dos resultados depois de feita a exponencial de y foi realizada da seguinte maneira: i) Coeficientes maiores que 1: considera-se que houve um aumento no número de dias de consumo de lácteos informais, retirando-se do valor do coeficiente com transformação exponencial uma unidade e multiplicando-o por 100 para saber a porcentagem de aumento. ii) Coeficientes menores que 1: considera-se que houve uma redução no número de dias de consumo de lácteos informais, fazendo-se a subtração 1 menos o valor do coeficiente com transformação exponencial e multiplicando-o por 100 (Hair Júnior et al., 2009 ).

O software OpenEpi (Dean, et al.,2011 b) foi usado para a regressão logística estratificada e para a regressão linear e avaliação de seu ajuste foi utilizado o software R (Development Core Team, 2005).

\section{Resultados e discussão}

A Tabela 1 apresenta as características descritivas das variáveis qualitativas (categóricas). A população total do estudo foi de 189 entrevistados. Destes, 139 (73,5\%) eram do sexo masculino. Entre os entrevistados, seis $(3,2 \%)$ moravam atualmente no meio rural, $54(28,6 \%)$ eram ex-moradores e $73(38,6 \%)$ nunca moraram no meio rural. Do total de entrevistados, 59 (31,2\%) já trabalhavam e $73(38,6 \%)$ nunca trabalhavam com pecuária ou agroindústria de alimentos de origem animal. Dos 189, $58(30,7 \%)$ eram consumidores atuais e $51(27,0 \%)$ eram exconsumidores de queijos feitos de leite cru.

A taxa de consumo atual $(30,7 \%)$ de queijo feito com leite cru do presente estudo foi menor que a de outro estudo $(66,5 \%)$ realizado por Silva et al., (2005) em bairros da periferia de Juiz de Fora. Entretanto, foi encontrada no presente estudo também uma taxa expressiva de ex-consumidores de queijo não pasteurizado (38,6 \%).

A Tabela 2 apresenta as características descritivas das variáveis quantitativas. A idade mínima e a mediana dos participantes foram de 17 anos e 39 anos, respectivamente. A variável renda familiar apresentou valor mediano de 1,9 salário mínimo. A quantidade de consumo de queijo não pasteurizado ao longo da vida apresentou um valor mediano de 279 unidades.

Este estudo produziu estimativas da prevalência de consumo, bem como das quantidades de queijo feito com leite cru ao longo da vida. Adicionalmente, modelos explicativos univariado e multivariado para a quantidade de consumo de queijo feito com leite cru ao longo da vida foram avaliados.
Tabela 1: Características descritivas da população do estudo ( $n=189)$, Juiz de Fora, 2008-2010 (variáveis qualitativas)

\begin{tabular}{|c|c|}
\hline Característica & $\mathrm{n}(\%)$ \\
\hline $\begin{array}{l}\text { Sexo } \\
\text { Masculino } \\
\text { Feminino }\end{array}$ & $\begin{array}{c}139(73,5) \\
50(26,5)\end{array}$ \\
\hline $\begin{array}{l}\text { Cor da pele } \\
\text { Branco } \\
\text { Negro ou pardo } \\
\text { Ignorado }\end{array}$ & $\begin{array}{l}48(25,4) \\
67(34,4) \\
74(40,2)\end{array}$ \\
\hline $\begin{array}{l}\text { Nível de Escolaridade } \\
\text { Nenhuma até a } 4^{\mathrm{a}} \text { série ensino fundamental } \\
5^{\mathrm{a}} \text { a } 8^{\mathrm{a}} \text { série ensino fundamental } \\
\text { Ensino médio incompleto até superior completo } \\
\text { Ignorado }\end{array}$ & $\begin{array}{l}61(32,3) \\
53(28,0) \\
31(16,0) \\
44(23,7)\end{array}$ \\
\hline $\begin{array}{l}\text { Local de residência } \\
\text { Juiz de Fora } \\
\text { Outros municípios }\end{array}$ & $\begin{array}{c}161(85,2) \\
28(14,8)\end{array}$ \\
\hline $\begin{array}{l}\text { Histórico de residência no meio rural } \\
\text { Moradores atuais } \\
\text { Ex-moradores } \\
\text { Nunca moraram } \\
\text { Ignorado }\end{array}$ & $\begin{array}{c}6(3,2) \\
54(28,6) \\
73(38,6) \\
56(29,6)\end{array}$ \\
\hline
\end{tabular}

\section{Tipo de moradia}

Barraco/Quarto/Presídio/Albergue/Hospital Psiquiátrico $\quad 22(11,6)$

Casa/Apartamento $118(62,4)$

Ignorado

$49(26,0)$

\section{Ocupação}

Trabalham com pecuária ou agroindústria de alimentos de origem animal

Não trabalham com as atividades acima

Ignorado

\section{Estado civil}

Solteiro

Desquitado/Divorciado

$12(6,3)$

Casado/Viúvo

$5(2,6)$

Ignorado

$90(47,7)$

\section{Estado conjugal}

Nunca morou com parceiro sexual $\quad 24(12,8)$

Já morou com parceiro sexual $\quad 53(28,0)$

$56(29,6)$

Ignorado

$56(29,6)$

\section{Histórico do consumo de álcool no último ano}

Beberam de forma exagerada

Nunca bebeu ou não bebeu no último ano

Histórico do consumo de álcool em binge no último ano

Binge de uma a mais vezes por semana

$38(20,1)$

Binge de uma vez ao mês a uma vez ao ano

Nunca bebeu binge no último ano

Nunca bebeu ou nunca bebeu em binge

Ignorado

\section{Diagnóstico sorológico para HIV}

Positivo

Ignorados

\section{Histórico do consumo de queijo feito com leite cru}

Consumidores atuais

Ex-consumidores

$58(30,7 \%)$

Nunca consumiram

$51(27 \%)$

Ignorados 
Tabela 2: Características descritivas da população do estudo ( $n=189)$, Juiz de Fora, 2008-2010 (Variáveis quantitativas)

\begin{tabular}{ccccccc}
\hline Característica & $\begin{array}{c}\text { Valor } \\
\text { mínimo }\end{array}$ & $\mathbf{2 5 \%}$ & Mediana & $\mathbf{7 5 \%}$ & $\begin{array}{c}\text { Valor } \\
\text { Máximo }\end{array}$ & Moda \\
\hline Idade & 17,0 & 30,0 & 39,0 & 50,0 & 83,0 & 25,0 \\
\hline Renda Individual & 0,0 & 0,3 & 1,0 & 1,3 & 12,9 & 0,0 \\
\hline $\begin{array}{c}\text { Renda Familiar } \\
\text { Histórico do }\end{array}$ & 0,0 & 1,0 & 1,9 & 2,8 & 24,0 & 1,0 \\
$\begin{array}{c}\text { consumo de queijo } \\
\text { feito com leite cru }\end{array}$ & 0,0 & 9,0 & 279,0 & 1272,0 & 21840,0 & 0,0 \\
\hline
\end{tabular}

A Tabela 3 apresenta resultados da análise univariada de regressão linear para o consumo de queijo não pasteurizado ao longo da vida. As variáveis idade, sexo, ocupação relacionada com a pecuária ou agroindústria de alimentos de origem animal e histórico de residência no meio rural apresentaram associação $(p \leq 0,05)$ com o número de dias de consumo de queijo feito de leite cru ao longo da vida.

Tabela 3: Análise univariada para o consumo de queijo feito com leite cru longo da vida

\begin{tabular}{|c|c|c|c|}
\hline Variável & $\begin{array}{l}\text { Coeficiente } \\
\text { (LnY) }\end{array}$ & Coeficiente & Valor $p$ \\
\hline Idade & 0,04 & 1,04 & $s^{*}$ \\
\hline Sexo (masculino/feminino) & 2,61 & 13,61 & $\mathrm{~S}^{* * *}$ \\
\hline $\begin{array}{l}\text { Ocupação pecuária ou agroindústria } \\
\text { (trabalha/não trabalha) }\end{array}$ & 2,80 & 16,60 & $\mathrm{~S}^{* * *}$ \\
\hline $\begin{array}{l}\text { Residência meio rural } \\
\text { (moram ou já moraram/nunca moraram) }\end{array}$ & 2,18 & 8,90 & $\mathrm{~S}^{\star * *}$ \\
\hline $\begin{array}{l}\text { Local de residência } \\
\text { (outros municípios/Juiz de Fora) }\end{array}$ & $-1,69$ & 0,18 & ns \\
\hline Moradia (casa ou apartamento/outros) & 0,87 & 2,39 & ns \\
\hline Renda familiar & $-0,01$ & 0,98 & ns \\
\hline $\begin{array}{l}\text { Escolaridade }\left(1^{\mathrm{a}} \text { a } 4^{\mathrm{a}} \text { série }\right) \\
5^{\mathrm{a}} \text { a } 8^{\mathrm{a}} \text { série } \\
\text { médio a superior }\end{array}$ & $\begin{array}{l}0,02 \\
0,12\end{array}$ & $\begin{array}{l}1,02 \\
1,13\end{array}$ & $\begin{array}{l}\text { ns } \\
\text { ns }\end{array}$ \\
\hline $\begin{array}{l}\text { Bebida (nunca bebeu ou não no último ano) } \\
\text { moderado } \\
\text { exagerado }\end{array}$ & $\begin{array}{l}-0,36 \\
-1,09\end{array}$ & $\begin{array}{l}0,69 \\
0,33\end{array}$ & $\begin{array}{l}\text { ns } \\
\text { ns }\end{array}$ \\
\hline
\end{tabular}

ns= não significativo; s= significativo; " $=p<0,01 ; "=p<0,05 ;{ }^{* * *}=p<0,001$

A Tabela 4 apresenta análise multivariada de regressão linear para a quantidade de consumo de queijo feito com leite cru ao longo da vida. As variáveis ocupação e sexo apresentaram associação ( $p \leq 0,05)$ com o consumo deste alimento. Pessoas do sexo masculino apresentaram 7,92 vezes o número de dias de consumo das pessoas do sexo feminino, mostrando que pessoas do sexo masculino tiveram $692,01 \%$ a mais de consumo. Com relação à ocupação, aqueles envolvidos com as relacionadas com a pecuária ou agroindústria de alimentos de origem animal apresentaram 10,87 vezes o número de dias de consumo de pessoas que tinham outras ocupações, um aumento de $987,43 \%$.

No modelo de regressão linear multivariada algumas variáveis que não apresentaram associação significativa com o consumo de queijo feito de leite cru permaneceram nos modelos para melhor ajuste. A variável ocupação com pecuária ou agroindústria de produtos de origem animal apresentou associação com o consumo de queijo feito com leite cru. Possivelmente porque estas ocupações facilitam o consumo devido à proximidade do consumidor com a fabricação ou comercialização destes produtos, ou de ser influenciado pela cultura comum no meio rural de que o produto lácteo cru é mais gostoso e saudável.

A variável sexo foi associada ao consumo de queijo feito de leite cru, estando o sexo masculino relacionado com maiores quantidades de consumo. Em contraposição, estudos realizados em duas cidades brasileiras (Jacareí e Colatina) concluíram que o consumo de derivados lácteos informais ocorre independentemente do sexo (Sousa, 2005; Miller, 2008).

O consumo de leite cru e derivados feitos com leite cru causa inúmeros prejuízos à saúde da população, porém esses dados não são, na maioria das vezes, divulgados. O problema é que estes produtos podem oferecer riscos à saúde humana, uma vez que muitos patógenos são veiculados pelo leite (Mycobacteirum bovis, Brucella spp., Campylobacter jejuni, Escherichia coli, entre outros micro-organismos com potencial risco à saúde).

O conhecimento dos principais patógenos existentes no leite cru, desde as etapas iniciais de produção, é de extrema importância para a Saúde Pública, uma vez que, a partir desses dados, é possível a criação de políticas de controle de doenças causadas por esses agentes. O mais preocupante é que o alimento contaminado com patógenos para humanos, geralmente não apresenta nenhuma alteração visível (de cheiro, gosto ou aparência), sendo as pessoas, em geral, afetadas sem perceberem. A criação de políticas e programas de conscientização quanto ao consumo de leite cru e derivados

Tabela 4: Análise multivariada para o consumo de queijo feito com leite cru ao longo da vida

\begin{tabular}{lcccc}
\hline \multicolumn{1}{c}{ Variável } & $\begin{array}{c}\text { Coeficiente } \\
\text { (Ln Y) }\end{array}$ & Coeficiente & $\begin{array}{c}\text { Impacto } \\
\text { (\%) }\end{array}$ & Valor $\boldsymbol{p}$ \\
\hline Idade & 0,02 & 1,02 & - & $\mathrm{ns}$ \\
\hline $\begin{array}{l}\text { Sexo } \\
\text { (Masculino/Feminino) }\end{array}$ & 2,06 & 7,92 & 692,01 & $\mathrm{~s}^{* * * *}$ \\
$\begin{array}{l}\text { Ocupação pecuária ou agroindústria } \\
\text { (trabalha/não trabalha) }\end{array}$ & 2,38 & 10,87 & 987,43 & $\mathrm{~s}$ \\
\hline Renda familiar & 0,05 & 1,05 & - & $\mathrm{ns}$ \\
\hline
\end{tabular}

ns= não significativo; $s=$ significativo; ${ }^{*}=p<0,01 ; "=p<0,05 ;{ }^{* * *}=p<0,001$ 
feitos com leite cru é uma ferramenta primordial para o controle das doenças transmitidas por alimentos (DTA'S) e combate ao comércio ilegal destes produtos tanto em municípios de pequeno porte quanto em grandes centros.

Considerando-se o negligenciamento do tipo de inspeção sanitária e/ou desconhecimento do nível de controle higiênicosanitário dos animais que estão envolvidos com a produção do tipo de leite referido no presente estudo, assim como de seus processos de obtenção, transporte e comercialização, os achados do presente estudo constituem um motivo de preocupação, principalmente em função do risco representado pelo consumo deste produto sem que tenha sido previamente submetido à fervura.

Deve ser ainda ressaltado que entre os 189 pacientes com tuberculose do presente estudo foram encontradas três evidências de tuberculose causada por $M$. bovis, sendo dois deles consumidores atuais de queijos feitos com leite cru, um ex-consumidor deste tipo de queijo (Silva et al., 2013).

Os resultados de informalidade do consumo de queijo feito com leite cru verificados no presente estudo e as variáveis a ela associadas, bem como os casos de tuberculose zoonótica verificados na mesma população do estudo analisado por Silva et al. (2013) deveriam servir de alerta tanto para as autoridades de saúde como da agricultura.

Esse estudo apresenta algumas limitações que devem ser discutidas. Os dados de prevalência e quantidade de consumo de queijo feito de leite cru, por terem sido levantados em uma população restrita (pacientes com tuberculose) tem uma validade interna alta, mas provavelmente uma baixa validade externa. Pacientes com tuberculose em geral possuem alta vulnerabilidade social, expressas por menores rendas e baixos níveis educacionais. Essas características talvez possam levar a maiores taxas de consumo de queijos feitos de leite cru que na população geral (queijos informais em geral são mais baratos). A análise dos fatores associados, por outro lado, embora tenham sido também conduzidos na mesma população, utilizou regressão linear multivariada, portanto, avaliou o efeito independente de cada possível variável explicativa sobre a variável resposta (quantidade de consumo de queijo feito de leite cru). Assim, os resultados dos fatores associados ao consumo de queijo feito de leite cru, por terem passado por uma análise multivariada, são mais passíveis de extrapolação que os dados de prevalência/quantidade de consumo.

\section{Conclusões}

Conclui-se com este estudo que sexo e ocupação relacionada com a pecuária ou agroindústria de alimentos de origem animal apresentam-se associados ao consumo de queijo feito com leite cru, também denominado informal, explicando, assim, a relevante taxa encontrada no consumo deste produto nesse estudo, alertando autoridades de saúde e agropecuária sobre o risco do consumo deste produto informal.

\section{Agradecimentos}

À Embrapa Gado de Leite pelo apoio na execução do projeto, à Coordenação de Aperfeiçoamento de Pessoal de Nível Superior pela bolsa de mestrado concedida a ROCHA, BB e ao Conselho Nacional de Desenvolvimento Científico e Tecnológico (suporte financeiro CNPq - 410595/2006-3/MCT/CNPq/MS-SCTIEDECIT 25/2006) pelos recursos destinados ao projeto. Finalmente, ao CNPq pela bolsa de produtividade concedida à MOREIRA, MAS.

\section{Referências}

DEAN, A.G.; ARNER, T.G.; SUNKI, G.G.; FRIEDMAN, R.; LANTINGA, M.; SANGAM, S. et al. Epi Info ${ }^{\mathrm{TM}}$, a database and statistics program for public health professionals. CDC, Atlanta, GA, USA, 2011 a.

DEAN, A.G.; SULLIVAN, K.M,.; SOE, M.M. OpenEpi: Open Source Epidemiologic Statistics for Public Health, Version 2.3.1. June, 2011 b. Disponível: www.OpenEpi.com

DEVELOPMENT CORE TEAM R: a language and environment for statistical computing. R Foundation for Statistical Computing, Vienna, Austria. 2005 [cited 2014 May 21]. http://www.R-project. org.

FERNANDES, R.F. Segurança alimentar: percepção e atitudes de consumidores de áreas periurbanas de Juiz de Fora - MG. Rev Hig Alimentar v.19, n. 130, p 1-2, 2005.

GUIMARÃES, F.F.; LANGONI, H. Leite: alimento imprescindível, mas com riscos para a saúde pública. Vet Zootec, v. 16, p. 38-51, 2009.

HAIR JÚNIOR, J.F.;BLACK, W.C.; BABIN, B.J.; ANDERSON, R.E.; TATHAM, R.L. Análise multivariada de dados. 6. ed. Porto Alegre: Bookman, 2009.

MILLER, N.B. Perfil do consumo de leite e derivados lácteos no município de Colatina - Espírito Santo. Dissertação (Especialização lato sensu): Universidade Castelo Branco, 2008.
RUBEZ, J. Consumo de leite cru traz riscos para a saúde. Feed \& Food 2012. http://www.feedfood.com.br/consumo-de-leite-crutraz-riscos-para-a-saude (acessado em 23/Maio/2013).

SILVA, M.R.; OLIVEIRA, M.E.; OLIVEIRA, L.D.; RODRIGUES, V.B.;

SILVA, M.R.; ROCHA, A.S.; COSTA, R.R.; ALENCAR, A.P.; OLIVEIRA, V.M.; JÚNIOR, A.A.F. et al. Tuberculosis in patients co-infected wint Mycobacterium bovis and Mycobacterium tuberculosis in an urban area of Brazil. Mem Inst Oswaldo Cruz, v. 106, p. 321-327, 2013.

SOUSA, D.D.P. Consumo de produtos lácteos informais em Jacareí-SP. São Paulo, 2005. 114 f. Dissertação (Mestrado). Departamento de Medicina Veterinária Preventiva e Saúde Animal, Universidade de São Paulo, 2005.

TENÓRIO, T.G.S.; MELO, L.E.H.; MOTA, R.A.; FERNANDES, C.H.C.; SÁ, L.M.; SOUTO, R.J.C.; et al. Pesquisa de fatores de risco para a brucelose humana associados à presença de brucelose bovina no município de Correntes, estado de Pernambuco, Brasil. Arq Inst Biol v. 75, n. 4, p. 415-421, 2008.

ZOCCAL, R. Leite inspecionado no Brasil 2000/2011. Embrapa Gado de Leite. Fevereiro 2012. http://www.cnpgl.embrapa.br/ (acessado em 15/Agosto/20013). 